\title{
Phase Controlled Piezoelectric-Transformer Backlight Inverter with No Magnetic Device
}

\author{
Wei-Chuan $\mathrm{Su} \quad$ Po-Chen Lin Chern-Lin Chen \\ Department of Electrical Engineering \\ \& Graduate Institute of Electronics Engineering \\ National Taiwan University \\ Taipei, Taiwan 10617
}

\begin{abstract}
This paper presents a CCFL backlight inverter which uses the quasi-modal piezoelectric transformer. The control loop locks the phase difference between input voltage and output current of piezoelectric transformer directly. It can approach the maximum-gain frequency within a range of load resistance value with proper locking phase.
\end{abstract}

\section{INTRODUCTION}

With the advantages of small size and high power density, piezoelectric transformer (PT) is attractive to the low power converting applications. The most important one is the backlight inverter for cold cathode fluorescent lamp (CCFL), because of the thickness requirement of LCD monitor and LCDTV. Additionally, PT has other advantages over winding transformer, such as high efficiency, low electromagnetic noise and no fire hazard [1,2,3]. These features are suitable for the increasing length and numbers of CCFLs in the backlight model. However, the major drawback of the PT is the narrow bandwidth of its operating frequency. In order to maintain the operating point, a feedback loop is necessary to control a PT inverter. Reference [4] and [6] show that phase control strategy is used to maintain PT's efficiency.

An inductor is usually added in front of PT. This resonant inductor and input capacitance of PT function as a filter. It is because that both CCFL and PT require a sinusoidal driving voltage $[2,4,6]$. However, value of the inductor is crucial. Low inductance may filter nothing and high inductance will attenuate the input voltage [6]. The electromagnetic noise generated by the inductor is also against the advantage of PT. A quasi-modal actuator PT is introduced to solve the problem [7]. With quasi-modal actuator, PT will sink energy only from a specific operating frequency [9]. Since PT can be a filter with itself, the resonant inductor is unnecessary and the PT can be driven with square voltage directly.

This paper presents a control method for quasi-modal PT backlight inverter. It locks the phase difference between input voltage and output current of PT. However, CCFL has various equivalent resistances and it will affect the frequency response of PT [8]. As a result, different input voltages are applied to change the equivalent resistance of CCFL and find the proper phase difference.

\section{ANALYsis Of THE BACKLIGHT INVERTER System}

PT is a device with high Q factor and narrow bandwidth and a phase-controlled loop is required to maintain the operating point of PT. With the inductor, the PT is considered as resonating and the controlling phase could be changed with different inductance values [6]. However, with quasi-modal PT, the parameters of PT are fixed and a determined phase difference should be controlled.

Fig. 1(a) is the PT backlight inverter driven by half bridge circuit, and fig. 1(b) shows the equivalent circuit of this inverter. The quasi-modal piezoelectric transformer will filter the dc component and harmonic of the input square voltage,

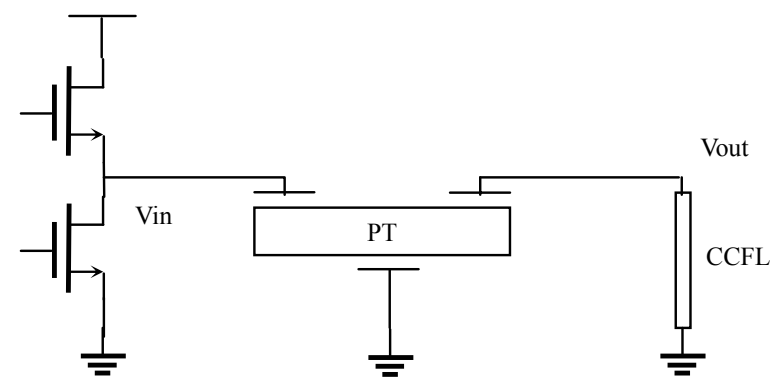

(a)

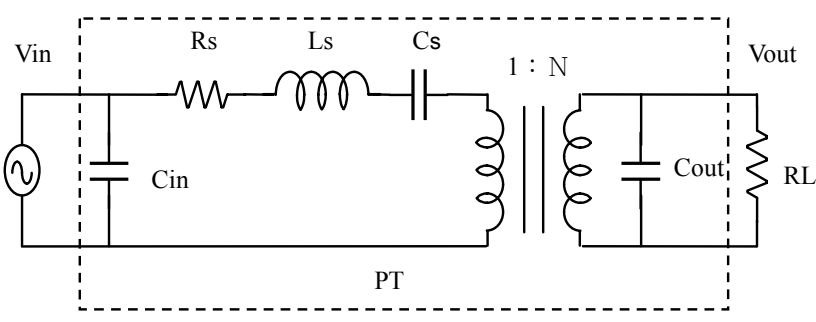

(b)

Fig. 1 (a) PT Backlight inverter, (b) Equivalent circuit of the backlight inverter 
so the input is replaced by a sinusoidal voltage source. The RL shows the load resistance of CCFL. And the PT is replaced by a widely adopted equivalent circuit.

According to fig. 1(b), the transfer function of this inverter can be shown as

$$
\frac{\text { Vout }}{\text { Vi }}=\frac{1}{N\left(R_{s}+j \omega L_{s}+\frac{1}{j \omega C_{s}}\right) \times\left(j \omega C_{\text {out }}+\frac{1}{R_{L}}\right)+\frac{1}{N}} .
$$

If RL can match to Cout at the resonant frequency of PT, the denominator of the transfer function will have minimum magnitude at that frequency. Besides, Rs is usually small, so PT will have maximum voltage gain and maximum power transfer at resonant frequency [9]. Then, the transfer function can be reduced to

$$
\frac{\text { Vout }}{\text { Vi }}=\frac{1}{\frac{1}{N} \times\left(j \omega C_{\text {out }} N^{2} R_{s}+\frac{N^{2} R_{s}+R_{L}}{R_{L}}\right)} .
$$

Since the equivalent resistance of CCFL is usually hundred kilo-ohms, which is much larger than $\mathrm{N}^{2} \mathrm{Rs}$, the phase difference between input voltage and output voltage will be nearly 45 degrees. However, CCFL changes its resistance with different brightness. With different RL, optimal operating frequency and phase would be changed.

Fig. 2 shows the simulation result of this inverter with different RL. Parameters of the experimented PT are shown below.

$$
\begin{array}{lll}
\text { Cin }=528.12 p F & \mathrm{Ls}=159.49 \mathrm{mH} & \mathrm{Cs}=48.25 \mathrm{pF} \\
\mathrm{Rs}=36.88 \Omega & \mathrm{Cout}=7.60 \mathrm{pF} & \mathrm{N}=6.05
\end{array}
$$

Cout and RL are converted to primary side for simulation, so the voltage gain is divided by $\mathrm{N}^{2}$.

According to the value of Cout, 350 kilo-ohms is the match resistance of RL. As a result, the resistance values near the match one are chosen for simulation. The simulation result shows that the optimal operating frequency of PT increases with the growing of its load resistance. However, PT can still maintain approximately the maximum gain when the output voltage delays input voltage 45 degrees. Since a CCFL has nearly the same driving voltage after igniting, the lamp current can be considered as the inverse of RL. And according to the simulation result, it can track the change of optimal operating frequency within a dimming range with 45 degrees phase lock control.

\section{Circuit Design}

\section{A. Block Diagram of Backlight Inverter System}

In order to fix the phase between input voltage and output voltage at 45 degrees, a Phase-Lock Loop (PLL) is applied. PLL is composed by voltage controlled oscillator (VCO), phase detector and low-pass filter (LPF). Two signals with the same frequency are fed into phase detector, and then the phase difference of the two signals is translated into voltage signal through LPF. Finally, the voltage controls the VCO that generates the operating frequency.

Fig. 3 shows the system of the backlight inverter. The

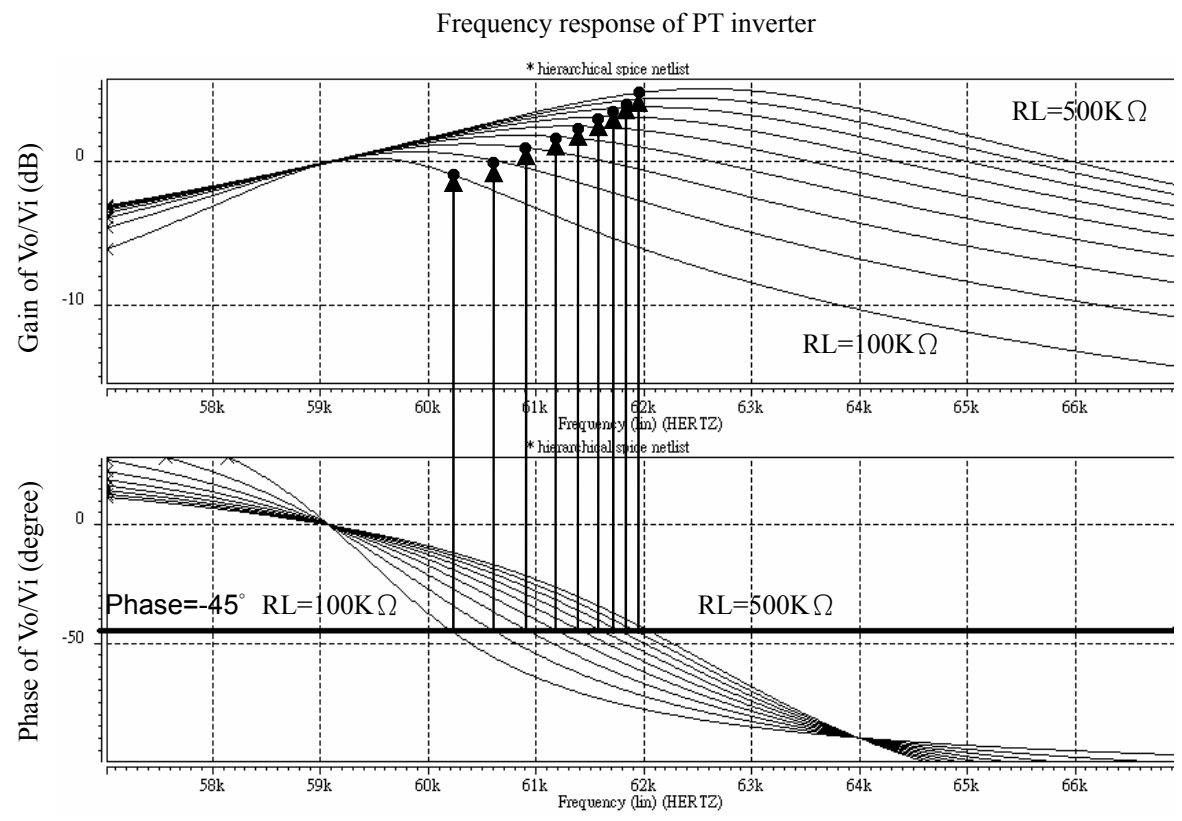

Fig. 2 Simulation of Gain and Phase of Vo/Vi with $\mathrm{RL}=100 \mathrm{k} \sim 500 \mathrm{k} \Omega$, Vo is divided by $\mathrm{N}^{2}$ 


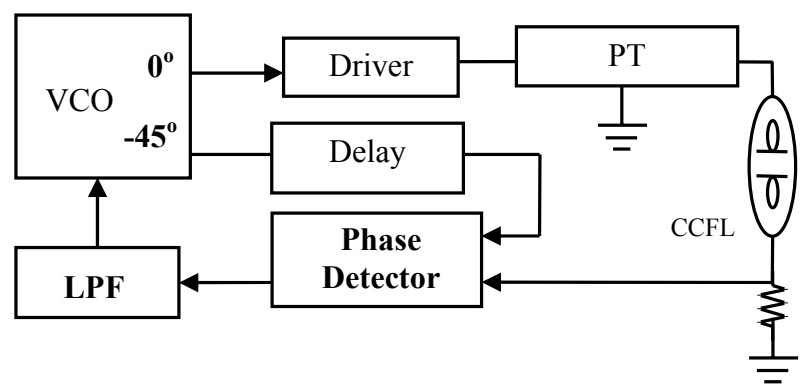

Fig. 3 Block Diagram of the Backlight Inverter

output voltage of PT is too high to be a feedback signal, so the lamp current, which is in-phase with lamp voltage, is chosen to be the feedback signal of the PLL. For the reference signal, it is much easier for phase detector to detect the signals in-phase, so the VCO is designed to generate two signals. The phase 0 degree signal drives the PT and the -45 degrees one is the feedback signal. By locking the lamp current and feedback signal in-phase, the input and output voltages of PT will maintain -45 degrees phase difference.

\section{B. Two Outputs Voltage Controlled Oscillator}

Fig. 4 shows the two outputs VCO. VCO4 is a VCO that has the operating frequency four times higher than the resonant frequency of PT. By adding an inverter, the time delay between A and B is half of the VCO4's period. After divided by two toggle $\mathrm{D}$ flip-flop, the delay is one eighth of the output period. It gives a fixed 45 degrees delay even with different frequency.

\section{Low-Pass filter and Phase Detector}

If the Phase difference is less than 45 degrees, the phase detector will discharge the LPF to increase the driving frequency, and vice versa. The phase detector is digital circuit switching at the operating frequency, which is the same as the output frequency of VCO. In order to block the switching noise from the control signal of $\mathrm{VCO}$, the $3 \mathrm{~dB}$ frequency of the LPF is about $5 \mathrm{kHz}$, which is less than $1 / 10$ of the operating frequency of the VCO and PT.

\section{Delay Circuit and Driver}

Fig. 5 shows a phase detector that has detecting range from minus 180 degrees to 180 degrees. The driver is a half bridge circuit formed by 2 power NMOSs and its driving circuit. And a delay circuit is added to compensate the delay of driver. Moreover, the delay circuit has another function. Since the frequency response of PT changes sharply near the resonant frequency, it would be easier using phase as the variance to measure the response of circuit. It can be also used to measure the change of the optimal locking phase with different load resistance.

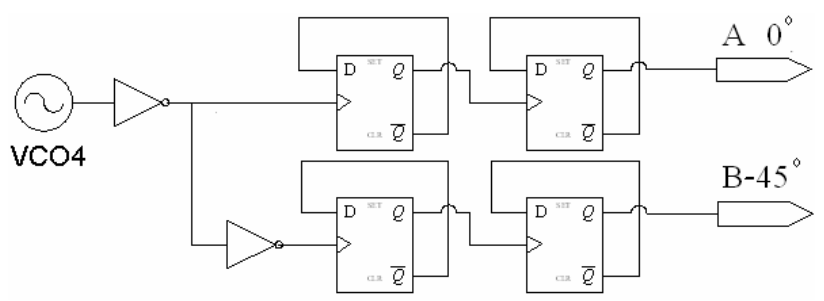

Fig. 4 VCO with 0 degree and -45 degrees Outputs

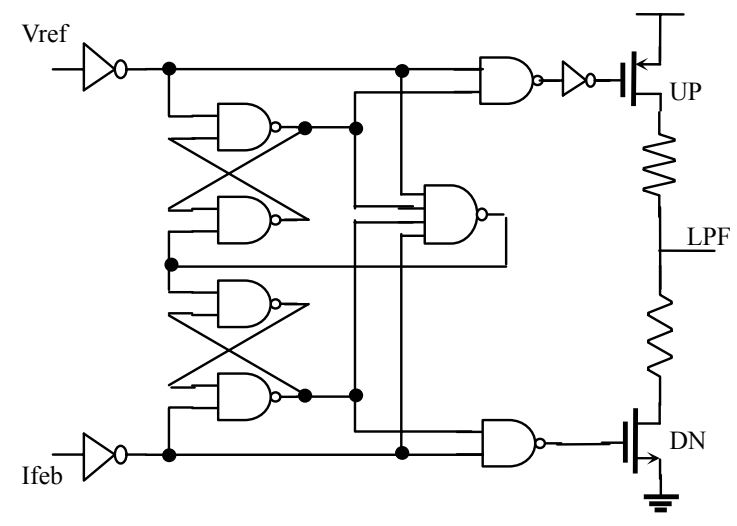

Fig. 5 -180 degrees $\sim 180$ degrees phase detector

\section{EXPERIMENTAL RESULTS}

A $10 \mathrm{~cm}$ CCFL is driven by this backlight inverter. Because of the negative resistance property, driving voltage of CCFL changes slightly. As a result, the voltage gain is almost constant for a given input driving voltage. However, with different locking phase, the CCFL has different current and the equivalent load resistance changes. There would be a maximum lamp current for a given voltage gain, and it is equivalent to the maximum voltage gain of that load resistance.

Fig. 6 is some waveforms of the backlight inverter system. Fig. 6(a) shows the output signal of $\mathrm{VCO} 4$, driving voltage of PT and the lamp current. Signal of VCO4 has various periods. It is because that VCO4 is affected by the switching noise of the half bridge circuit. It may change the phase difference between two signals of VCO. However, it could be compensated by the delay circuit. Fig 6(b) shows the reference signal, driving voltage of PT and the lamp current. The delay circuit is workable and the reference signal lags driving signal by 45 degrees. Fig. 6(c) shows driving voltage of PT, the lamp current and the lamp voltage. Phase difference between lamp current and driving signal is about -45 degrees. Driving voltage of CCFL is about $1000 \mathrm{Vp}-\mathrm{p}$. The operating frequency is about $60 \mathrm{kHz}$. 


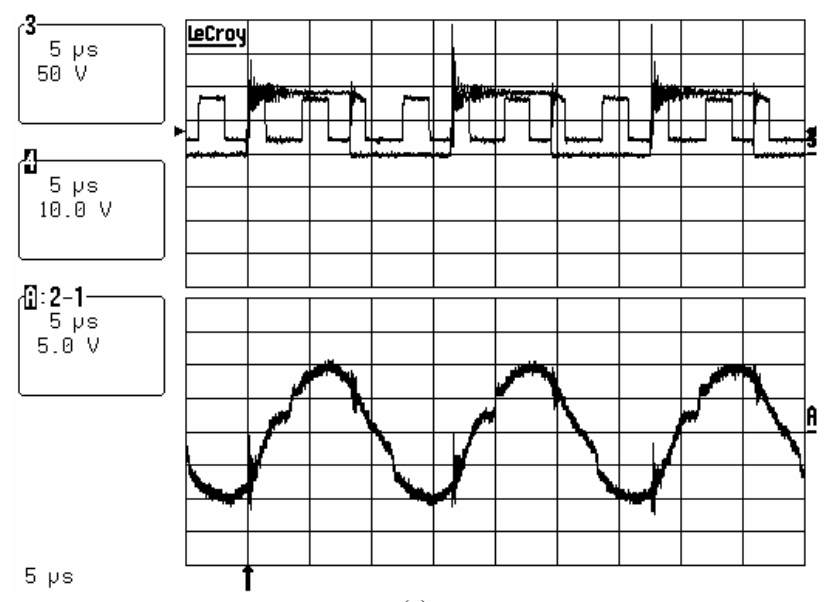

(a)

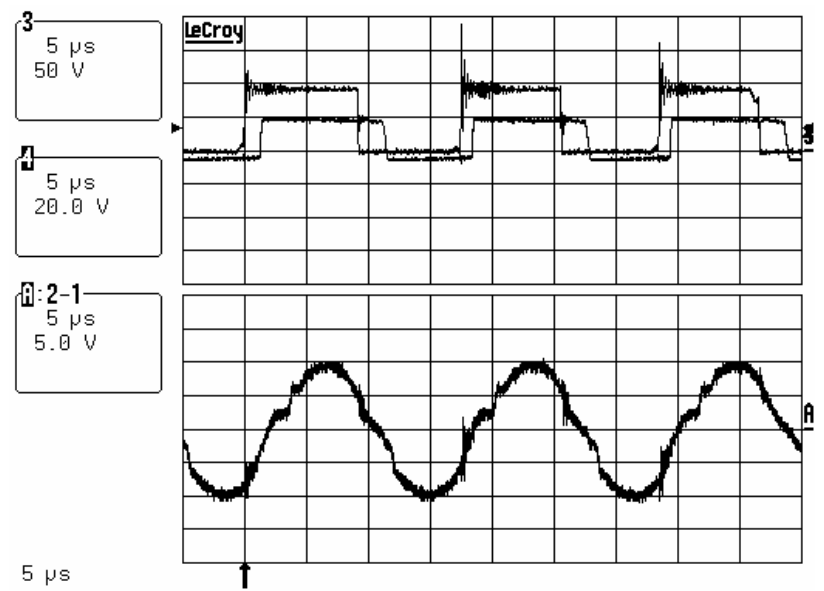

(b)

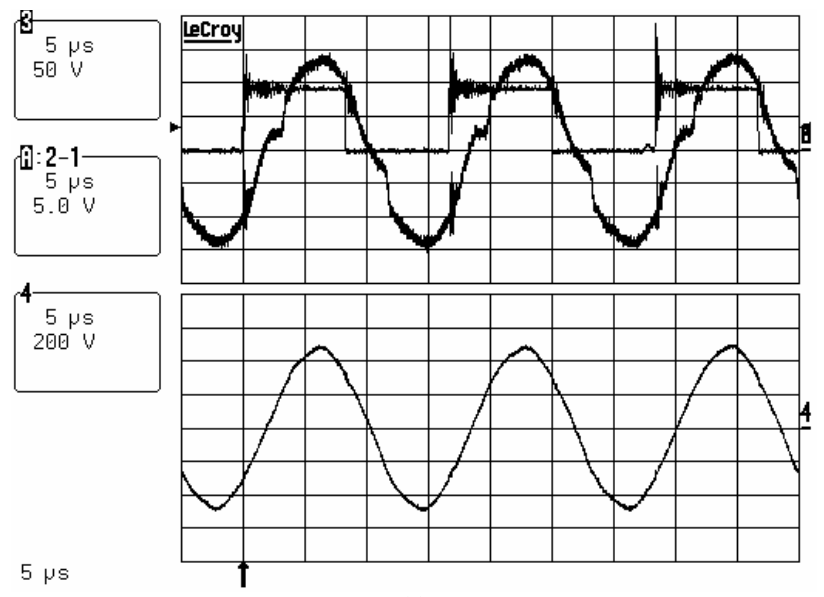

(c)

Fig. 6 Waveforms of (a) input voltage of PT, output voltage of VCO4 and lamp current, (b) input voltage of PT, reference signal and lamp current, (c) input voltage of PT, lamp current and lamp voltage.

By changing the locking phase, fig. 7 shows the phase versus lamp current with different input voltage. The flat

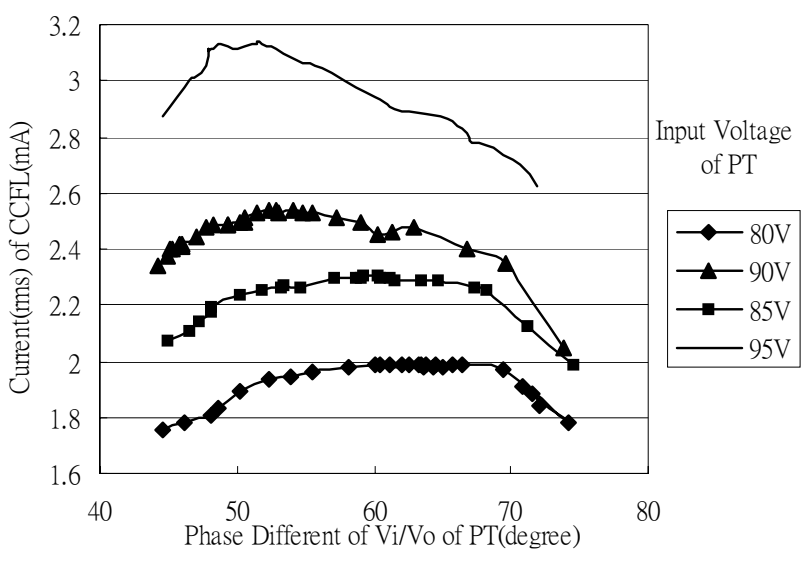

Fig. 7 Phase difference between input voltage and output current versus lamp current.

response and the overlap of the flat region show the possibility to define a constant locking phase over a range of input voltage. It also shows that the higher load resistance has higher voltage gain, which is the same as simulation result. However, the values of phase difference are larger than the simulation results. According to the output capacitance, the match resistance is 350 kilo-ohms. The equivalent resistances of CCFL at the maximum current are 171 kilo-ohms for input voltage is $80 \mathrm{~V}, 147$ kilo-ohms for $85 \mathrm{~V}, 134$ kilo-ohms for $90 \mathrm{~V}$, and 109 kilo-ohms for $95 \mathrm{~V}$ with $340 \mathrm{Vrms}$ lamp voltage. All of them have an optimal phase larger than simulation result.

\section{CONCLUSIONS}

In this paper, a control strategy for the backlight inverter using quasi-modal PT is presented. The phase difference between input voltage and the output current of PT is locked directly, so the resonant inductor can be saved. By controlling the delay circuit inserted in PLL, the relationship between PT's gain and phase shift can be measured much clear. And according to the experiment result, 55 degrees may be a proper phase difference. The PT can be operated around the optimal frequency with load current ranging from 2 to 3 milli-Amps. The acceptable range of load current makes this method suitable for CCFL's with various resistance values or even for dimming control.

\section{REFERENCE}

[1]. H. Kakehashi, T. Hidaka, T. Ninomiya, M. Shoyama, H. Ogasawara, Y. Ohta, "Electronic ballast using piezoelectric transformers for fluorescent lamps," IEEE PESC Vol.1, pp.29 - 351998.

[2]. G. Spiazzi, S. Buso, P. Tomasin, "Cold cathode fluorescent lamp power supply based on piezoelectric transformers," PESC 04. 2004 IEEE 35th Annual Volume 1, 20-25 June 2004 Page(s):406 - 412

[3]. R.L. Lin, F.C. Lee, E.M. Baker, D.Y. Chen, "Inductor-less 
piezoelectric transformer electronic ballast for linear fluorescent lamp," IEEE APEC, Vol.2, pp.664 - 669, 2001

[4]. S. Nakashima, T. Ninomiya, H. Ogasawara, H. Kakehashi, "Piezoelectric-transformer inverter with maximum-efficiency tracking and dimming control," IEEE APEC, Vol.2, pp.918 - 923, 2002.

[5]. C.S. Moo, W.M. Chen, H.K. Hsieh, "Electronic ballast with piezoelectric transformer for cold cathode fluorescent lamps," Electric Power Applications, IEE Proceedings, Vol.150, No. 3, pp.278 - 282, 2003.

[6]. C.H. Lin, Y. Lu, K.J. Pai, Y.Q. Chen, "Achieving maximum-efficiency tracking control for backlight electronic ballast with phase-locked loop techniques," IEEE International Conference on Control Applications, Vol.2, pp. 1651 - 1656, 2004.

[7]. C.K. Lee, W.H. Hsiao, C.W. Hsieh, "Piezoelectric transducer apparatus having a model-shaped electrode," U.S. Patent 6597085 B2. 2001.

[8]. C.G. Kim, K.C. Lee; B.H. Cho, "Modeling of CCFL using lamp delay and stability analysis of backlight inverter for large size LCD TV" IEEE APEC, Vol.3, pp. 1751 - 1757, 2005.

[9]. Y.H. Hsu, C.K. Lee, W.H. Hsiao, "Optimizing piezoelectric transformer for maximum power transfer" Smart Materials and Structures Vol.12, No. 3, pp.373-383, 2003. 